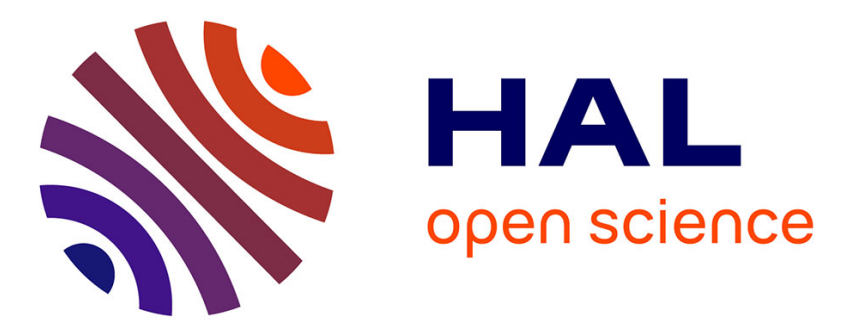

\title{
Positive invariance of polyhedrons and comparison of Markov reward models with different state spaces
}

Mourad Ahmane, James Ledoux, Laurent Truffet

\section{To cite this version:}

Mourad Ahmane, James Ledoux, Laurent Truffet. Positive invariance of polyhedrons and comparison of Markov reward models with different state spaces. Lecture notes in control and information sciences, 2006, 341, pp.153-160. hal-00447075

\section{HAL Id: hal-00447075 \\ https://hal.science/hal-00447075}

Submitted on 26 Aug 2013

HAL is a multi-disciplinary open access archive for the deposit and dissemination of scientific research documents, whether they are published or not. The documents may come from teaching and research institutions in France or abroad, or from public or private research centers.
L'archive ouverte pluridisciplinaire HAL, est destinée au dépôt et à la diffusion de documents scientifiques de niveau recherche, publiés ou non, émanant des établissements d'enseignement et de recherche français ou étrangers, des laboratoires publics ou privés. 


\title{
Positive invariance of polyhedrons and comparison of Markov reward models with different state spaces*
}

\author{
Mourad Ahmane ${ }^{1}$, James Ledoux ${ }^{2}$, and Laurent Truffet $^{3}$ \\ 1 IRCCyN, 1 rue de la Noë BP 92101, 44321 Nantes Cedex 3, France. \\ Mourad.Ahmane@irccyn.ec-nantes.fr \\ 2 INSA de Rennes \& IRMAR, 20 avenue des Buttes de Coesmes, CS 14315, 35043 \\ Rennes cedex, France. \\ James.Ledoux@insa-rennes.fr \\ 3 École des Mines de Nantes \& IRCCyN, 4 rue Alfred Kastler, BP 20722, 44307 \\ Nantes Cedex 3, France. \\ Laurent.Truffet@emn.fr
}

\begin{abstract}
In this paper, we discuss the comparison of expected rewards for discrete-time reward Markov chains with different state spaces. Necessary and sufficient conditions for such a comparison are derived. Due to the special nature of the introduced binary relation, a criterion may be formulated in terms of an inclusion of polyhedral sets. Then, algebraic and geometric forms are easily obtained from Haar's Lemma. Our results allow us to discuss some earlier results on the stochastic comparison of functions of Markov chains.
\end{abstract}

Key words: Linear discrete-time systems; Invariant sets; Haar's lemma.

\section{Basic notation}

- For all $m \in \mathbb{N}^{*}, \leq_{m}$ is the component-wise ordering of $\mathbb{R}^{m}$.

- Vectors are column vectors. ()$^{\top}$ is the standard transpose operator.

- $\mathbf{1}_{k}\left(\right.$ resp. $\mathbf{0}_{k}$ ) denotes the $k$-dimensional vector with all components equal to 1 (resp. 0).

- $\forall k \geq 1$, the set $\mathcal{S}_{k}$ denotes the set of all $k$-dimensional stochastic vectors.

- The set of real (resp. non-negative) $k \times n$-matrices is denoted by $\mathcal{M}_{k, n}(\mathbb{R})$ (resp. $\left.\mathcal{M}_{k, n}\left(\mathbb{R}_{+}\right)\right)$. $\mathbf{0}_{m, k}$ is the element of $\mathcal{M}_{m, k}(\mathbb{R})$ with all coefficients equal to $0 . \boldsymbol{I}_{k}$ is the identity element of $\mathcal{M}_{k, k}(\mathbb{R})$.

- If $\boldsymbol{G}_{1} \in \mathcal{M}_{m, n_{1}}(\mathbb{R}), \boldsymbol{G}_{2} \in \mathcal{M}_{m, n_{2}}(\mathbb{R})$, then $\left(\boldsymbol{G}_{1} \mid \boldsymbol{G}_{2}\right) \in \mathcal{M}_{m, n_{1}+n_{2}}(\mathbb{R})$.

* Partially supported by the MATHSTIC CNRS project entitled Contrôle de systèmes à événements discrets via des techniques de comparaison et de réduction de systèmes stochastiques 
- If $\boldsymbol{A}, \boldsymbol{B} \in \mathcal{M}_{m, n}(\mathbb{R})$ then $\boldsymbol{A} \leq \boldsymbol{B}$ denotes the entry-wise comparison of the matrices $\boldsymbol{A}$ and $\boldsymbol{B}$.

\section{Introduction}

A discrete-time autonomous linear state-space model is the basic model in control theory specified by

$$
\left\{\begin{aligned}
\boldsymbol{x}(0) \in \mathbb{R}^{d}, \boldsymbol{x}(n+1) & =\boldsymbol{A} \boldsymbol{x}(n), n \in \mathbb{N} \\
\boldsymbol{o}_{x}(n) & =\boldsymbol{R} \boldsymbol{x}(n) .
\end{aligned}\right.
$$

where $\boldsymbol{A} \in \mathcal{M}_{d, d}(\mathbb{R})$ and $\boldsymbol{R} \in \mathcal{M}_{l, d}(\mathbb{R})$. $(\boldsymbol{x}(n))$ is the sequence of states of the system and $\left(\boldsymbol{o}_{x}(n)\right)$ the sequence of outputs. Such a system is said to be positive [1] if, for any $\boldsymbol{x}(0) \in \mathbb{R}^{d}$, we have

$$
\mathbf{0}_{d} \leq_{d} \boldsymbol{x}(0) \Longrightarrow \forall n \in \mathbb{N}, \mathbf{0}_{d} \leq_{d} \boldsymbol{x}(n) \text { and } \mathbf{0}_{l} \leq_{l} \boldsymbol{o}_{x}(n) .
$$

Then, it is easily seen that the autonomous linear state-space model (1) is positive iff $\mathbf{0}_{d, d} \leq \boldsymbol{A}$ and $\mathbf{0}_{l, d} \leq \boldsymbol{R}$. We refer to [1] for a detailed account for the theory and applications of such models. We only deal here with positive autonomous linear state-space model for which $\boldsymbol{A}$ is a stochastic matrix. Specifically, the Markov reward models are considered.

A Markov reward model is a very general stochastic model. It is used, for instance, in assessing the performability of systems [2] which is a measure combining dependability and performance aspects of a system. Let us recall the basic setup for such a class of models. We consider a $\mathscr{E}$-valued Markov chain $(X(n))$ where $\mathscr{E}$ is a finite set and $\boldsymbol{A}$ its transition probabilities matrix, i.e. $\forall i, j \in \mathscr{E}, A_{j, i}=P(X(n+1)=j \mid X(n)=i)$. Suppose that $(X(n))$ is a Markovian model of some system. Now, a cost or reward $r_{i} \geq 0$ is associated with each visit of $(X(n))$ to the state $i \in \mathscr{E}$. Then, the random variable $r_{X(n)}$ is the instantaneous reward "gained" at time $n$. The $d$-dimensional non-negative vector $\boldsymbol{r}$ corresponding to the family of rewards $\left\{r_{i} \mid i \in \mathscr{E}\right\}$ is often related to a specific management/maintenance policy. With this simple Markov reward model, we associate the autonomous linear state-space model specified by

$$
\left(d, \boldsymbol{A}, 1, \boldsymbol{r}^{\top}\right):\left\{\begin{aligned}
\boldsymbol{x}(0) \in \mathcal{S}_{d}, \boldsymbol{x}(n+1) & =\boldsymbol{A} \boldsymbol{x}(n), \quad n \in \mathbb{N} \\
o_{x}(n) & =\boldsymbol{r}^{\top} \boldsymbol{x}(n) .
\end{aligned}\right.
$$

The scalar output $o_{x}(n)$ is the expected reward at time $n$ for the vector of rewards $\boldsymbol{r}$. Then, we can be interested in comparing two policies which are identified to the two vectors $\boldsymbol{r}$ and $\boldsymbol{r}^{\prime}$. We define two autonomous linear statespace models $\left(d, \boldsymbol{A}, 1, \boldsymbol{r}^{\top}\right)$ and $\left(d, \boldsymbol{A}, 1, \boldsymbol{r}^{\boldsymbol{\prime}^{\top}}\right)$ as in (2). We can compare their output sequences through the usual ordering on $\mathbb{R}$. A slightly more general situation is the comparison of two different weightings of the same family of policies. Indeed, let us identify the policies $\boldsymbol{r}_{1}, \boldsymbol{r}_{2}, \ldots, \boldsymbol{r}_{l}$ to a $l \times d$-matrix $\boldsymbol{R}$. 
We consider two vectors of weights : $\boldsymbol{c}=\left(c_{1}, \ldots, c_{l}\right)$ and $\boldsymbol{c}^{\prime}=\left(c_{1}^{\prime}, \ldots, c_{l}^{\prime}\right)$. The following autonomous linear state-space model is defined by

$$
(d, \boldsymbol{A}, l, \boldsymbol{R}):\left\{\begin{aligned}
\boldsymbol{x}(0) \in \mathcal{S}_{d}, \boldsymbol{x}(n+1) & =\boldsymbol{A} \boldsymbol{x}(n), \quad n \in \mathbb{N} \\
\boldsymbol{o}_{x}(n) & =\boldsymbol{R} \boldsymbol{x}(n) .
\end{aligned}\right.
$$

The vector $\boldsymbol{o}_{x}(n)$ is the vector of expected reward "gained" at time $n$ according to the different policies of "rewarding". Then, we analyze the output sequence $\left(\boldsymbol{o}_{x}(n)\right)$ via

$$
\boldsymbol{c}^{\top} \boldsymbol{o}_{x}(n) \leq \boldsymbol{c}^{\prime \top} \boldsymbol{o}_{x}(n) .
$$

The two previous situations is easily adapted to the case where the underlying Markov chains are different, that is we compare two different Markov models. Thus, we can be interested in comparing various models of the same system through their corresponding maintenance policies. In other words, we deal with a decision making problem in a context of multi-criteria and multi-models of a system. Therefore, the general comparison of the output sequences of (multi-dimensional) Markov reward models should be investigated. This paper proposes criteria for two such sequences to be comparable with respect to the binary relation defined, for any $(\boldsymbol{x}, \boldsymbol{y}) \in \mathbb{R}^{l} \times \mathbb{R}^{l^{\prime}}$, by

$$
\boldsymbol{x} \underset{\boldsymbol{C}, \boldsymbol{C}^{\prime}}{\leq} \boldsymbol{y} \stackrel{\text { def }}{\Longleftrightarrow} \boldsymbol{C} \boldsymbol{x} \leq_{m} \boldsymbol{C}^{\prime} \boldsymbol{y}
$$

where $\boldsymbol{C} \in \mathcal{M}_{m, l}(\mathbb{R})$ and $\boldsymbol{C}^{\prime} \in \mathcal{M}_{m, l^{\prime}}(\mathbb{R})$. This binary relation should be thought of as an abstract version of an integral stochastic order for discrete random variables (e.g. see [3, Chap 2]). Let $\left(\boldsymbol{o}_{x}(n)\right),\left(\boldsymbol{o}_{y}(n)\right)$ be the output sequences of the systems $(d, \boldsymbol{A}, l, \boldsymbol{R})$ and $\left(d^{\prime}, \boldsymbol{A}^{\prime}, l^{\prime}, \boldsymbol{R}^{\prime}\right)$ as defined in (3). We investigate the conditions under which the assertion: $\forall \boldsymbol{x}(0) \in \mathbb{R}^{d}, \forall \boldsymbol{y}(0) \in \mathbb{R}^{d^{\prime}}$

$$
\left(\boldsymbol{x}(0) \in \mathcal{S}_{d}, \boldsymbol{y}(0) \in \mathcal{S}_{d^{\prime}}, \boldsymbol{o}_{x}(0) \underset{\boldsymbol{C}, \boldsymbol{C}^{\prime}}{\leq} \boldsymbol{o}_{y}(0)\right) \Longrightarrow \forall n \in \mathbb{N}, \boldsymbol{o}_{x}(n) \underset{\boldsymbol{C , \boldsymbol { C } ^ { \prime }}}{\leq} \boldsymbol{o}_{y}(n)
$$

is true. In this case, the two Markov reward models $(d, \boldsymbol{A}, l, \boldsymbol{R})$ and $\left(d^{\prime}, \boldsymbol{A}^{\prime}, l^{\prime}, \boldsymbol{R}^{\prime}\right)$ are said to be $\left(\boldsymbol{C}, \boldsymbol{C}^{\prime}\right)$-comparable.

The paper is organized as follows. Useful results on the polyhedrons and polyhedral sets inclusion are recalled below. In Section 2, necessary and sufficient conditions for (5) to be true are provided. In Section 3, the comparison of functions of Markov chains and related works are discussed. We conclude in Section 4.

The basic materials to our approach is now reported.

\section{Inclusion of polyhedrons and invariance sets}

The polyhedron associated with $\boldsymbol{P} \in \mathcal{M}_{m, k}(\mathbb{R})$ and $\boldsymbol{p} \in \mathbb{R}^{m}$ is defined by

$$
\mathcal{P}(\boldsymbol{P}, \boldsymbol{p}):=\left\{\boldsymbol{x} \in \mathbb{R}^{k} \mid \boldsymbol{P} \boldsymbol{x} \leq_{m} \boldsymbol{p}\right\} .
$$


Recall that $\mathcal{S}_{k}, \mathcal{S}_{k} \times \mathcal{S}_{k^{\prime}}$ are polyhedrons and the intersect of two polyhedrons is a polyhedron.

We restate a result proved by Haar [4] that provides an algebraic characterization of the inclusion of two polyhedral sets.

Lemma 1 (Haar). Let $\boldsymbol{P} \in \mathcal{M}_{m, k}(\mathbb{R}), \boldsymbol{Q} \in \mathcal{M}_{r, k}(\mathbb{R})$ and $\boldsymbol{p} \in \mathbb{R}^{m}, \boldsymbol{q} \in \mathbb{R}^{r}$. If $\mathcal{P}(\boldsymbol{P}, \boldsymbol{p}) \neq \emptyset$ then

$$
\mathcal{P}(\boldsymbol{P}, \boldsymbol{p}) \subseteq \mathcal{P}(\boldsymbol{Q}, \boldsymbol{q}) \Longleftrightarrow \exists \boldsymbol{H} \in \mathcal{M}_{r, m}\left(\mathbb{R}_{+}\right): \boldsymbol{Q}=\boldsymbol{H} \boldsymbol{P} \text { and } \boldsymbol{H} \boldsymbol{p} \leq_{r} \boldsymbol{q} .
$$

Now, we recall the connection between the inclusion of polyhedrons and the invariant sets. A reference for such material is [5] for instance.

Definition 1. A subset $\mathcal{V}$ of $\mathbb{R}^{k}$ is said to be positively invariant under $M \in$ $\mathcal{M}_{m, k}(\mathbb{R})$ if $\boldsymbol{M} \mathcal{V} \subseteq \mathcal{V}$, where $\boldsymbol{M V}:=\{\boldsymbol{M} \boldsymbol{x} \mid \boldsymbol{x} \in \mathcal{V}\}$.

The following corollary of Haar's lemma is our basic tool for deriving our results.

Lemma 2. Let $\boldsymbol{P} \in \mathcal{M}_{m, k}(\mathbb{R}), \boldsymbol{M} \in \mathcal{M}_{k, k}(\mathbb{R})$ and $\boldsymbol{p}, \boldsymbol{q} \in \mathbb{R}^{m}$. If $\mathcal{P}(\boldsymbol{P}, \boldsymbol{p}) \neq \emptyset$, then we have

$$
\begin{aligned}
\boldsymbol{M P}(\boldsymbol{P}, \boldsymbol{p}) \subseteq \mathcal{P}(\boldsymbol{P}, \boldsymbol{q}) & \Longleftrightarrow \mathcal{P}(\boldsymbol{P}, \boldsymbol{p}) \subseteq \mathcal{P}(\boldsymbol{P} \boldsymbol{M}, \boldsymbol{q}) \\
& \Longleftrightarrow \exists \boldsymbol{H} \in \mathcal{M}_{m, m}\left(\mathbb{R}_{+}\right): \boldsymbol{P} \boldsymbol{M}=\boldsymbol{H} \boldsymbol{P} \text { and } \boldsymbol{H p} \leq_{m} \boldsymbol{q} .
\end{aligned}
$$

\section{Comparison of multi-dimensional Markov reward models}

Let $\mathscr{E}$ and $\mathscr{F}$ be two finite sets with respective cardinal $d$ and $d^{\prime}$. Introduce two Markov chains $(X(n))$ and $(Y(n))$ with respective state spaces $\mathscr{E}, \mathscr{F}$ and transition probabilities matrices $\boldsymbol{A}$ and $\boldsymbol{A}^{\prime}$. The probability distribution of the random variable $X(n)$ (resp. $Y(n))$ is denoted by $\boldsymbol{x}(n)$ (resp. $\boldsymbol{y}(n)$ ). Let us consider the corresponding autonomous linear state-space models $(d, \boldsymbol{A}, l, \boldsymbol{R})$ and $\left(d^{\prime}, \boldsymbol{A}^{\prime}, l^{\prime}, \boldsymbol{R}^{\prime}\right)$ as in (3). The $l \times d$ (resp. $\left.l^{\prime} \times d^{\prime}\right)$ matrix $\boldsymbol{R}$ (resp. $\left.\boldsymbol{R}^{\prime}\right)$ is called the rewards matrix associated with $(X(n))$ (resp. $(Y(n))$ ). The autonomous linear state-space model $(d, \boldsymbol{A}, l, \boldsymbol{R})$ is called a multi-dimensional Markov reward model associated with the Markov chain $(X(n)$ ) (or the stochastic matrix $\boldsymbol{A}$ ) and the rewards matrix $\boldsymbol{R}$. The output $\boldsymbol{o}_{x}(n)$ (resp. $\boldsymbol{o}_{y}(n)$ ) is the multi-dimensional expected reward at time $n$. Note that the sequence $\left(\boldsymbol{o}_{x}(n)\right)$ does not have a linear dynamics in general.

Define $\boldsymbol{R S}_{d}:=\left\{\boldsymbol{R} \boldsymbol{x}, \boldsymbol{x} \in \mathcal{S}_{d}\right\}$ and $\boldsymbol{R}^{\prime} \mathcal{S}_{d^{\prime}}:=\left\{\boldsymbol{R}^{\prime} \boldsymbol{y}, \boldsymbol{y} \in \mathcal{S}_{d^{\prime}}\right\}$. Let us introduce the following condition for $C \in \mathcal{M}_{m, l}(\mathbb{R})$ and $\boldsymbol{C}^{\prime} \in \mathcal{M}_{m, l^{\prime}}(\mathbb{R})$ :

$$
\mathrm{H}:\left\{(\boldsymbol{x}, \boldsymbol{y}) \in \boldsymbol{R} \mathcal{S}_{d} \times \boldsymbol{R}^{\prime} \mathcal{S}_{d^{\prime}} \mid \boldsymbol{C} \boldsymbol{x} \leq_{m} \boldsymbol{C}^{\prime} \boldsymbol{y}\right\} \neq \emptyset .
$$

Now, the main result of this section is proved by applying Lemma 2 to 


$$
\boldsymbol{P}:=\left(\begin{array}{cc}
\boldsymbol{C} \boldsymbol{R} & -\boldsymbol{C}^{\prime} \boldsymbol{R}^{\prime} \\
-\boldsymbol{I}_{d} & \mathbf{0}_{d, d^{\prime}} \\
\mathbf{0}_{d^{\prime}, d}, & -\boldsymbol{I}_{d^{\prime}} \\
\mathbf{1}_{d}^{T} & \mathbf{0}_{d^{\prime}}^{T} \\
-\mathbf{1}_{d}^{T} & \mathbf{0}_{d^{\prime}}^{T} \\
\mathbf{0}_{d}^{T} & \mathbf{1}_{d^{\prime}}^{T} \\
\mathbf{0}_{d}^{T} & -\mathbf{1}_{d^{\prime}}^{T}
\end{array}\right), \boldsymbol{p}:=\left(\begin{array}{c}
\mathbf{0}_{m+d+d^{\prime}} \\
1 \\
-1 \\
1 \\
-1
\end{array}\right)
$$

and the following stochastic matrix as matrix $\boldsymbol{M}: \boldsymbol{M}\left(\boldsymbol{A}, \boldsymbol{A}^{\prime}\right):=$ $\left(\begin{array}{cc}\boldsymbol{A} & \mathbf{0}_{d, d^{\prime}} \\ \mathbf{0}_{d^{\prime}, d} & \boldsymbol{A}^{\prime}\end{array}\right)$. We omit the details for saving space.

Theorem 1. Assume $H$. The multi-dimensional Markov reward models $(d, \boldsymbol{A}, l, \boldsymbol{R})$ and $\left(d^{\prime}, \boldsymbol{A}^{\prime}, l^{\prime}, \boldsymbol{R}^{\prime}\right)$ are said to be $\left(\boldsymbol{C}, \boldsymbol{C}^{\prime}\right)$-comparable iff any of the following conditions is satisfied:

1. $\mathcal{P}\left(\left(\boldsymbol{C R} \mid-\boldsymbol{C}^{\prime} \boldsymbol{R}^{\prime}\right), \mathbf{0}_{m}\right) \cap \mathcal{S}_{d} \times \mathcal{S}_{d^{\prime}} \subseteq \mathcal{P}\left(\left(\boldsymbol{C R} \boldsymbol{A} \mid-\boldsymbol{C}^{\prime} \boldsymbol{R}^{\prime} \boldsymbol{A}^{\prime}\right), \mathbf{0}_{m}\right) \cap \mathcal{S}_{d} \times \mathcal{S}_{d^{\prime}}$.

2. Geometric criterion. The polyhedron $\mathcal{P}\left(\left(\boldsymbol{C R} \mid-\boldsymbol{C}^{\prime} \boldsymbol{R}^{\prime}\right), \mathbf{0}_{m}\right) \cap \mathcal{S}_{d} \times \mathcal{S}_{d^{\prime}}$ is positively invariant under the matrix $\boldsymbol{M}\left(\boldsymbol{A}, \boldsymbol{A}^{\prime}\right)$.

3. Algebraic criterion. There exist $\boldsymbol{H} \in \mathcal{M}_{m, m}\left(\mathbb{R}_{+}\right)$and vectors $\boldsymbol{u}, \boldsymbol{v} \in$ $\mathbb{R}^{m}$ such that

$$
-u \mathbf{1}_{d}^{\top} \leq \boldsymbol{H C R}-\boldsymbol{C R A}, \quad \boldsymbol{H} C^{\prime} \boldsymbol{R}^{\prime}-\boldsymbol{C}^{\prime} \boldsymbol{R}^{\prime} \boldsymbol{A}^{\prime} \leq \boldsymbol{v} \mathbf{1}_{d^{\prime}}^{\top}, \quad \boldsymbol{u}+\boldsymbol{v} \leq_{m} \mathbf{0}_{m} .
$$

The monotonicity property of a multi-dimensional Markov reward model is now introduced from (5).

Definition 2. A multi-dimensional Markov reward model $(d, \boldsymbol{A}, l, \boldsymbol{R})$ is said to be $\left(\boldsymbol{C}, \boldsymbol{C}^{\prime}\right)$-monotone when we have, for any $\boldsymbol{x}_{1}(0), \boldsymbol{x}_{2}(0) \in \mathbb{R}^{d}$,

$$
\left(\boldsymbol{x}_{1}(0), \boldsymbol{x}_{2}(0) \in \mathcal{S}_{d}, \boldsymbol{o}_{x_{1}}(0) \underset{\boldsymbol{C}, \boldsymbol{C}^{\prime}}{\leq} \boldsymbol{o}_{x_{2}}(0)\right) \Longrightarrow \forall n \in \mathbb{N}, \boldsymbol{o}_{x_{1}}(n) \underset{\boldsymbol{C}, \boldsymbol{C}^{\prime}}{\leq} \boldsymbol{o}_{x_{2}}(n) .
$$

The following criteria for the $\left(\boldsymbol{C}, \boldsymbol{C}^{\prime}\right)$-monotonicity of the Markov reward model $(d, \boldsymbol{A}, l, \boldsymbol{R})$ is deduced from Theorem 1 with $\boldsymbol{A}=\boldsymbol{A}^{\prime}$.

Corollary 1. Assume H. The multi-dimensional Markov reward model $(d, \boldsymbol{A}, l, \boldsymbol{R})$ is $\left(\boldsymbol{C}, \boldsymbol{C}^{\prime}\right)$-monotone iff any of the following conditions is satisfied:

1. $\mathcal{P}\left(\left(\boldsymbol{C R} \mid-\boldsymbol{C}^{\prime} \boldsymbol{R}\right), \mathbf{0}_{m}\right) \cap \mathcal{S}_{d} \times \mathcal{S}_{d^{\prime}} \subseteq \mathcal{P}\left(\left(\boldsymbol{C R A} \mid-\boldsymbol{C}^{\prime} \boldsymbol{R A}\right), \mathbf{0}_{m}\right) \cap \mathcal{S}_{d} \times \mathcal{S}_{d^{\prime}}$.

2. Geometric criterion. The polyhedron $\mathcal{P}\left(\left(\boldsymbol{C R} \mid-\boldsymbol{C}^{\prime} \boldsymbol{R}\right), \mathbf{0}_{m}\right) \cap \mathcal{S}_{d} \times \mathcal{S}_{d^{\prime}}$ is positively invariant under the matrix $\boldsymbol{M}(\boldsymbol{A}, \boldsymbol{A})$.

3. Algebraic criterion. There exist $\boldsymbol{H} \in \mathcal{M}_{m, m}\left(\mathbb{R}_{+}\right)$and vectors $\boldsymbol{u}, \boldsymbol{v} \in$ $\mathbb{R}^{m}$ such that

$$
-\boldsymbol{u} \mathbf{1}_{d}^{\top} \leq \boldsymbol{H C R}-\boldsymbol{C R A}, \quad H C^{\prime} \boldsymbol{R}-\boldsymbol{C}^{\prime} \boldsymbol{R A} \leq \boldsymbol{v} \mathbf{1}_{d}^{\top}, \quad \boldsymbol{u}+\boldsymbol{v} \leq_{m} \mathbf{0}_{m} .
$$

These results provide comparison conditions of the marginal distributions of functions of Markov chains. Thus, earlier results on stochastic comparison of functions of Markov chains is discussed below. 


\section{Functions of Markov chains}

Let $(X(n))$ and $(Y(n))$ be two Markov chains with respective state spaces $\mathscr{E}:=$ $\left\{e_{1}, \ldots, e_{d}\right\}, \mathscr{F}:=\left\{f_{1}, \ldots f_{d^{\prime}}\right\}$ and transition probabilities matrices $\boldsymbol{A}, \boldsymbol{A}^{\prime}$. Let $\mathscr{G}, \mathscr{G}^{\prime}$ be the finite sets $\left\{g_{1}, \ldots, g_{l}\right\}$ and $\left\{g_{1}^{\prime}, \ldots, g_{l^{\prime}}^{\prime}\right\}$ respectively. Consider two maps $\varphi: \mathscr{E} \rightarrow \mathscr{G}$ and $\psi: \mathscr{F} \rightarrow \mathscr{G}^{\prime}$. The matrices $\boldsymbol{R}$ and $\boldsymbol{R}^{\prime}$ are specified as follows

$$
\begin{aligned}
& \boldsymbol{R}(j, k):=1 \text { if } \varphi\left(e_{k}\right)=g_{j} \text { and } \boldsymbol{R}(j, k):=0 \text { otherwise } \\
& \boldsymbol{R}^{\prime}(j, k):=1 \text { if } \psi\left(f_{k}\right)=g_{j}^{\prime} \text { and } \boldsymbol{R}^{\prime}(j, k):=0 \text { otherwise. }
\end{aligned}
$$

The processes $(\varphi(X(n)))$ and $(\psi(Y(n)))$ are said to be (deterministic) functions of Markov chains. The sequence of outputs $\left(\boldsymbol{o}_{x}(n)\right)$ does not have a linear dynamics in general. Conditions on the matrix $\boldsymbol{A}$ under which $\left(\boldsymbol{o}_{x}(n)\right)$ also follows a linear dynamics may be found in [6]. Since $\boldsymbol{R}$ is stochastic, note that the condition $\mathrm{H}$ has the form $\mathcal{P}\left(\left(\boldsymbol{C} \mid-\boldsymbol{C}^{\prime}\right), \mathbf{0}_{m}\right) \neq \emptyset$.

The stochastic comparison of one-dimensional distributions of functions of Markov chains is well-known. This kind of problem is motivated for instance by performance evaluation and/or optimization of telecommunications networks (e.g. see [7]), by reliability assessment (e.g. see [8]). It also appears when we look for Markovian bounds on the output stream of a queuing network (e.g. see[9]). Some contributions on the comparison of the one-dimensional distributions of functions of Markov chains are the following. We refer to the original reference for the full details.

\section{Doisy's work.}

Doisy studied the comparison of functions of Markov chains using a coupling technique [8]. The setting is as follows

- $\mathscr{G}=\mathscr{G}^{\prime}$ (and thus $l=l^{\prime}$ ) and there exists a partial order $\prec$ on $\mathscr{G}$;

- $\boldsymbol{C}=\boldsymbol{C}^{\prime}=\boldsymbol{U}_{s t}^{\mathscr{G}} \in \mathcal{M}_{m, l}(\mathbb{R})$, where $\boldsymbol{U}_{s t}^{\mathscr{G}}$ denotes the matrix associated with the so-called strong ordering based on the partial order $\prec$ defined on $\mathscr{G}$. It means that each row of matrix $\boldsymbol{U}_{s t}^{\mathscr{G}}$ corresponds to the indicator function of an $\prec$-increasing subset of $\mathscr{G}$. Recall that a $\prec$-increasing set $\gamma \subseteq \mathscr{G}$ is defined by: $\gamma:=\left\{g \in \mathscr{G}: \forall g^{\prime} \in \mathscr{G}\left(g \prec g^{\prime} \Rightarrow g^{\prime} \in \gamma\right)\right\}$. Let us note that when $\prec$ is a total order then $\boldsymbol{U}_{s t}^{\mathscr{G}}$ is the $l \times l$ matrix defined by: $\boldsymbol{U}_{s t}^{\mathscr{G}}:=\left[1_{\left\{g \prec g^{\prime}\right\}}\right]_{g, g^{\prime} \in \mathscr{G}}$ where $1_{\{\cdot\}}$ is the indicator function of the set $\{\cdot\}$ (see also Kijima [10, Chap 3 and references therein]).

- two maps $\varphi: \mathscr{E} \rightarrow \mathscr{G}$ and $\psi: \mathscr{F} \rightarrow \mathscr{G}$ with associated matrices $\boldsymbol{R}$ and $\boldsymbol{R}^{\prime}$ defined in (8).

Doisy introduced the following definition of the $(\varphi, \psi)$-comparison of the two stochastic matrices $\boldsymbol{A}$ and $\boldsymbol{A}^{\prime}$ [8, Def 1, Section 3]:

$$
\boldsymbol{A} \leq_{\varphi, \psi} \boldsymbol{A}^{\prime} \Leftrightarrow\left(e_{i} \in \mathscr{E}, f_{j} \in \mathscr{F}, \varphi\left(e_{i}\right) \prec \psi\left(f_{j}\right) \Rightarrow \boldsymbol{R} \boldsymbol{A} \boldsymbol{\delta}_{i} \underset{\boldsymbol{U}_{s t}^{\varphi}, \boldsymbol{U}_{s t}^{\varphi g}}{\leq} \boldsymbol{R}^{\prime} \boldsymbol{A}^{\prime} \boldsymbol{\delta}_{j}^{\prime}\right),
$$


where $\left(\boldsymbol{\delta}_{i}\right)_{i=1, \ldots, d}$ and $\left(\boldsymbol{\delta}_{j}^{\prime}\right)_{j=1, \ldots, d^{\prime}}$ denote the canonical basis of $\mathbb{R}^{d}$ and $\mathbb{R}^{d^{\prime}}$, respectively. The strongest connected result of Doisy is reformulated as follows [8, Prop 2 and Th 4, Section 3].

Property 1. Let $(d, \boldsymbol{A}, l, \boldsymbol{R})$ and $\left(d^{\prime}, \boldsymbol{A}^{\prime}, l, \boldsymbol{R}^{\prime}\right)$ be two multi-dimensional Markov reward models as defined in (3). Then, $\boldsymbol{A} \leq_{\varphi, \psi} \boldsymbol{A}^{\prime}$ iff $(d, \boldsymbol{A}, l, \boldsymbol{R})$ and $\left(d^{\prime}, \boldsymbol{A}^{\prime}, l, \boldsymbol{R}^{\prime}\right)$ are $\left(\boldsymbol{U}_{s t}^{\mathscr{G}}, \boldsymbol{U}_{s t}^{\mathscr{G}}\right)$-comparable.

The "only if" part was algebraically proved using the definition of the $\left(\boldsymbol{U}_{s t}^{\mathscr{G}}, \boldsymbol{U}_{s t}^{\mathscr{G}}\right)$-comparability of $(d, \boldsymbol{A}, l, \boldsymbol{R})$ and $\left(d^{\prime}, \boldsymbol{A}^{\prime}, l, \boldsymbol{R}^{\prime}\right)$. Doisy's proof of the "if part" is based on the construction of a suitable coupling of the underlying Markov chains. An algebraic proof of the "if part" is provided from Theorem 1(3). Indeed, we know the following criterion for the $\left(\boldsymbol{U}_{s t}^{\mathscr{G}}, \boldsymbol{U}_{s t}^{\mathscr{G}}\right)$-comparison to hold: there exist $\boldsymbol{H} \in \mathcal{M}_{m, m}\left(\mathbb{R}_{+}\right)$and $\boldsymbol{u}, \boldsymbol{v} \in \mathbb{R}^{m}$ such that

$$
\begin{aligned}
-\boldsymbol{u} \mathbf{1}_{d}^{\top} \leq & \boldsymbol{H} \boldsymbol{U}_{s t}^{\mathscr{G}} \boldsymbol{R}-\boldsymbol{U}_{s t}^{\mathscr{G}} \boldsymbol{R} \boldsymbol{A} \\
& \boldsymbol{H} \boldsymbol{U}_{s t}^{\mathscr{G}} \boldsymbol{R}^{\prime}-\boldsymbol{U}_{s t}^{\mathscr{G}} \boldsymbol{R}^{\prime} \boldsymbol{A}^{\prime} \leq \boldsymbol{v} \mathbf{1}_{d^{\prime}}^{\top} \\
& \boldsymbol{u}+\boldsymbol{v} \leq_{m} \mathbf{0}_{m} .
\end{aligned}
$$

We must prove that properties (10a-10c) implies that $\boldsymbol{A} \leq_{\varphi, \psi} \boldsymbol{A}^{\prime}$. First, note that for any $e_{i} \in \mathscr{E}$ and $f_{j} \in \mathscr{F}$, we have

$$
\varphi\left(e_{i}\right) \prec \psi\left(f_{j}\right) \Longleftrightarrow \boldsymbol{R} \boldsymbol{\delta}_{i} \underset{U_{s t}^{\mathscr{S}}, \boldsymbol{U}_{s t}^{\mathscr{G}}}{\leq} \boldsymbol{R}^{\prime} \boldsymbol{\delta}_{j}^{\prime} \Longleftrightarrow \boldsymbol{U}_{s t}^{\mathscr{G}} \boldsymbol{R} \boldsymbol{\delta}_{i} \leq_{m} \boldsymbol{U}_{s t}^{\mathscr{G}} \boldsymbol{R}^{\prime} \boldsymbol{\delta}_{j}^{\prime}
$$

Since $\boldsymbol{H} \in \mathcal{M}_{m, m}\left(\mathbb{R}_{+}\right)$, we have

$$
\boldsymbol{H} \boldsymbol{U}_{s t}^{\mathscr{G}} \boldsymbol{R} \boldsymbol{\delta}_{i} \leq_{m} \boldsymbol{H} \boldsymbol{U}_{s t}^{\mathscr{G}} \boldsymbol{R}^{\prime} \boldsymbol{\delta}_{j}^{\prime} .
$$

Second, suppose that the conditions (10a-10c) hold. We find that

$$
\begin{aligned}
& \boldsymbol{U}_{s t}^{\mathscr{G}} \boldsymbol{R} \boldsymbol{A} \boldsymbol{\delta}_{i} \leq_{m} \boldsymbol{H} \boldsymbol{U}_{s t}^{\mathscr{G}} \boldsymbol{R} \boldsymbol{\delta}_{i}+\boldsymbol{u} \quad \text { from (10a) } \\
& \leq_{m} \boldsymbol{H} \boldsymbol{U}_{s t}^{\mathscr{G}} \boldsymbol{R} \boldsymbol{\delta}_{i}-\boldsymbol{v} \quad \text { from (10c) } \\
& \leq_{m} \boldsymbol{H} \boldsymbol{U}_{s t}^{\mathscr{G}} \boldsymbol{R}^{\prime} \boldsymbol{\delta}_{j}^{\prime}-\boldsymbol{v} \quad \text { from (11) } \\
& \leq_{m} \boldsymbol{U}_{s t}^{\mathscr{G}} \boldsymbol{R}^{\prime} \boldsymbol{A}^{\prime} \boldsymbol{\delta}_{j}^{\prime} \quad \text { from (10b). }
\end{aligned}
$$

Thus, we find from (9) that $\boldsymbol{A} \leq_{\varphi, \psi} \boldsymbol{A}^{\prime}$. The proof is complete.

The comparison of the finite-dimensional distributions of functions of Markov chains (also called in this context coupling condition) may be achieved from condition (9) due to a special property of the strong ordering.

\section{Abu-Amsha and Vincent's work.}

These authors considered the following setting in [11]:

- $\mathscr{E}$ is assumed to be totally ordered finite set; 
- $\mathscr{G}=\mathscr{G}^{\prime}=\mathscr{F}\left(\right.$ thus $\left.l=l^{\prime}=d^{\prime}\right)$;

- $\boldsymbol{R}^{\prime}=\boldsymbol{I}_{d^{\prime}}$, that is $\psi=i d$;

- $\boldsymbol{C}=\boldsymbol{C}^{\prime}$ (that implies $m=d=d^{\prime}$ ) is assumed to be invertible.

The authors founded the following sufficient condition for the Markov reward models $(d, \boldsymbol{A}, l, \boldsymbol{R})$ and $\left(d, \boldsymbol{A}^{\prime}, l, \boldsymbol{I}_{d}\right)$ to be comparable:

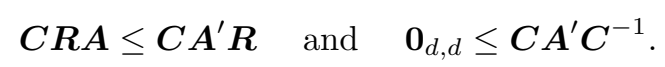

The conditions above are equivalent to : $\exists \boldsymbol{H} \in \mathcal{M}_{d, d}\left(\mathbb{R}_{+}\right)$such that

$$
C R A \leq C A^{\prime} R \quad \text { and } \quad C A^{\prime}=H C .
$$

It is easily checked that the algebraic criterion reported in Theorem 1 is fulfilled. However, it is also clear from Theorem 1 that these conditions are not necessary.

\section{Conclusion}

Criteria for the $\left(\boldsymbol{C}, \boldsymbol{C}^{\prime}\right)$-comparison of multi-dimensional Markov rewards are provided using Haar's lemma. In particular, this applied to the comparison of (deterministic) functions of Markov chains. Note that the probabilistic functions of Markov chains or the Hidden Markov models can also be considered. We do not go into further details here.

\section{References}

1. L. Farina and S. Rinaldi. Positive Linear Systems. Pure and Applid Mathematics. Wiley, 2000.

2. J. F. Meyer. On evaluating the performability of degradable computing systems. IEEE Trans. Comput., 29:3720-7313, 1980.

3. A. Muller and D. Stoyan. Comparison Methods for Stochastic Models and Risks. J. Wiley \& Sons, 2002.

4. A. Haar. Uber lineare ungleichungen (1918). In Reprinted in: A. Haar, Gesammelte Arbeiten. Akademi Kiado, 1959.

5. A. A. ten Dam and J. W. Nieuwenhuis. A linear programming algorithm for invariant polyhedral sets of discrete-time linear systems. Systems Control Lett., 25:337-341, 1995.

6. J. Ledoux. Linear dynamics for the state vector of Markov chain functions. Adv. in Appl. Probab., 36(4):1198-1211, 2004.

7. N. Pekergin. Stochastic performance bounds by state space reduction. Performance Evaluation, 36-37:1-17, 1999.

8. M. Doisy. A coupling technique for comparison of functions of Markov processes. J. Appl. Math. Decis. Sci., 4:39-64, 2000.

9. L. Truffet. Geometrical bounds on an output stream of a queue in atm switch: Application to the dimensioning problem. In ATM Networks: Performance Modelling and Analysis, Vol. II. Chapman-Hall, 1996.

10. M. Kijima. Markov Processes for Stochastic Modeling. Chapman-Hall, 1997.

11. O. Abu-Amsha and J.-M. Vincent. An algorithm to bound functionals of Markov chains with large state space. In 4th INFORMS Telecomm. Conf., Florida, 1998. 